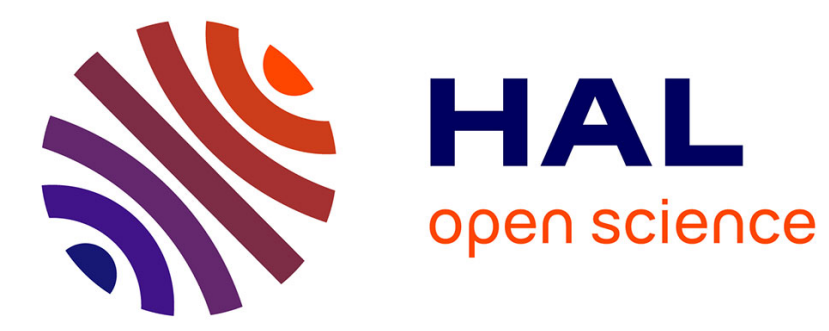

\title{
Approximate Joint Diagonalization within the Riemannian Geometry Framework
}

Florent Bouchard, Louis Korczowski, Jérôme Malick, Marco Congedo

\section{To cite this version:}

Florent Bouchard, Louis Korczowski, Jérôme Malick, Marco Congedo. Approximate Joint Diagonalization within the Riemannian Geometry Framework. EUSIPCO 2016 - 24th European Signal Processing Conference, Aug 2016, Budapest, Hungary. pp.210-214, 10.1109/EUSIPCO.2016.7760240 . hal-01370052

\section{HAL Id: hal-01370052 https://hal.science/hal-01370052}

Submitted on 21 Sep 2016

HAL is a multi-disciplinary open access archive for the deposit and dissemination of scientific research documents, whether they are published or not. The documents may come from teaching and research institutions in France or abroad, or from public or private research centers.
L'archive ouverte pluridisciplinaire HAL, est destinée au dépôt et à la diffusion de documents scientifiques de niveau recherche, publiés ou non, émanant des établissements d'enseignement et de recherche français ou étrangers, des laboratoires publics ou privés. 


\title{
Approximate Joint Diagonalization within the Riemannian Geometry Framework
}

\author{
Florent Bouchard*, Louis Korczowski*, Jérôme Malick ${ }^{\dagger}$, Marco Congedo* \\ *GIPSA-lab, CNRS, Univ. Grenoble Alpes, Grenoble Institute of Technology, Grenoble France \\ ${ }^{\dagger}$ LJK, CNRS, Univ. Grenoble Alpes, Grenoble France \\ Email: florent.bouchard@gipsa-lab.fr
}

\begin{abstract}
We consider the approximate joint diagonalization problem (AJD) related to the well known blind source separation (BSS) problem within the Riemannian geometry framework. We define a new manifold named special polar manifold equivalent to the set of full rank matrices with a unit determinant of their Gram matrix. The Riemannian trust-region optimization algorithm allows us to define a new method to solve the AJD problem. This method is compared to previously published NoJOB and UWEDGE algorithms by means of simulations and shows comparable performances. This Riemannian optimization approach thus shows promising results. Since it is also very flexible, it can be easily extended to block AJD or joint BSS.
\end{abstract}

Index Terms-approximate joint diagonalization; blind source separation; Riemannian geometry; Riemannian optimization; special polar manifold

\section{INTRODUCTION}

The approximate joint diagonalization (AJD) of a matrix set is instrumental to solve the well known blind source separation (BSS) problem [1]. It can be expressed as it follows: given a set of $K$ symmetric matrices $\left\{C_{k}\right\}_{1 \leq k \leq K}$ of dimension $n \times n$, find a full-rank matrix $B$ of dimension $n \times p$ (with $p \leq n$ ) such that the set $\left\{B^{T} C_{k} B\right\}_{1 \leq k \leq K}$ contains matrices as diagonal as possible according to some criterion.

A common diagonality criterion is the sum of squares of the off-diagonal elements of the transformed matrices leading to the minimization of the functional

$$
\mathrm{f}(B)=\sum_{k=1}^{K} \| \text { off }\left(B^{T} C_{k} B\right) \|_{F}^{2},
$$

where $B \in \mathbb{R}^{n p},\|\cdot\|_{F}$ denotes the Frobenius norm and off( $\left.\cdot\right)$ vanishes the diagonal elements of its argument.

While optimizing (1), we need to avoid the trivial solution $B=0$. Hence, some constraints on the joint diagonalizer are usually added. A common choice is to fix the norm of the columns of $B$ [2]-[4]. Another solution, when $B$ is square $(p=n)$, is to fix its determinant as proposed in [5]. In this paper, we generalize this latter constraint to the non-square case by fixing the determinant of the Gram matrix $B^{T} B$ of $B$.

Even though the AJD has already been extensively studied, proposed method are usually very specific to a cost function, a constraint and an optimization algorithm. Here, we propose a general framework based on Riemannian geometry that can handle various cost functions and optimization algorithms.
Furthermore, except those methods that seek an orthogonal diagonalizer [6] or that do not ensure full rank property [4], existing algorithms can only deal with square joint diagonalizer. If one wants to apply a dimension reduction in this case, a whitening step is mandatory [1], [7]. Such approach has been shown suboptimal [6]. Hence, to the best of our knowledge, we propose here the first method without severe limitations that can properly handle dimension reduction.

The aim of this paper is to show that it is possible to tackle the AJD problem within the Riemannian geometry framework. This allows to turn constrained optimization problems on embedded spaces into unconstrained problems on smooth manifolds [8]. The idea is to use the geometrical properties of the constraints to define a smooth manifold where the problem is not constrained anymore. This allows to handle constraints in a natural way. Riemannian approaches for AJD have already been considered for example in [4]-[6]. Moreover, a link between the Riemannian geometric mean of a set and its AJD has been recently found [9]. None of proposed Riemannian AJD methods suit well our purpose. They either make use of unadapted manifolds as in [4], [6] (see above) or only elements of Riemannian geometry are used and not the all framework as in [5]. In this article, we define an appropriate manifold for the AJD problem. In the following, we consider $p \leq n$. The manifold defined here is equivalent to the space $\mathcal{B}_{p, n}=\left\{B \in \mathbb{R}_{*}^{n p}: \operatorname{det}\left(B^{T} B\right)=1\right\}$ where $\mathbb{R}_{*}^{n p}$ is the set of full rank matrices and $\operatorname{det}($.$) denotes the determinant.$

This paper is divided into four sections including this introduction. In Section II-A, tools needed to perform optimization within the Riemannian framework are briefly introduced (see [8] for details). In Section II-B, The well-known Stiefel manifold [8] is presented. In Section II-C, a new manifold called special symmetric positive definite manifold is defined and its geometry is studied. The product of those two manifolds leads to a new manifold named special polar manifold in Section II-D. This latter manifold is shown to be equivalent to $\mathcal{B}_{p, n}$ defined above using the polar decomposition. In Section II-E, the objective function (1) is defined for the special polar manifold and its gradient and Hessian are derived. Then, the Riemannian trust-region method, which is chosen here as the optimization scheme, is presented in Section II-F. In Section III, the performance of the proposed method is analyzed and compared to state-of-the-art AJD algorithms [2], [3]. Finally, in Section IV, conclusions are drawn. 


\section{METHOD}

\section{A. Tools for Optimization on Riemannian Manifolds}

Riemannian optimization methods search for the next iterate in the tangent space of the current iterate using Riemannian differential geometry tools (Riemannian gradient, Riemannian Hessian). The solution in the tangent space is then projected back onto the manifold. In order to solve optimization problems within the Riemannian framework [8] one needs to define the following mathematical objects:

- the projection map that projects a point of the embedded space onto the tangent space at a given point of the manifold;

- a suitable Riemannian metric on the tangent space;

- the Levi Civita connection, which is essential to be able to define the Riemannian Hessian of a function;

- a retraction, which is a mapping from the tangent space back onto the manifold.

\section{B. Stiefel Manifold}

The Stiefel manifold $\mathrm{St}_{p, n}=\left\{U \in \mathbb{R}^{n p}: U^{T} U=I_{p}\right\}$ is well known. The reader is referred to [8] for proofs of the following:

$\mathrm{St}_{p, n}$ is an embedded submanifold of the Euclidean space $\mathbb{R}^{n p}$ of dimension $n p-\frac{1}{2} p(p+1)$. Its tangent space at $U$ is $T_{U} \mathrm{St}_{p, n}=\left\{\xi \in \mathbb{R}^{n p}: U^{T} \xi+\xi^{T} U=0\right\}$ endowed with the metric $\overline{\mathrm{g}}_{U}$ such that, for all $\xi$ and $\eta$ in $T_{U} \mathrm{St}_{p, n}$,

$$
\overline{\mathrm{g}}_{U}(\xi, \eta)=\operatorname{tr}\left(\xi^{T} \eta\right),
$$

where $\operatorname{tr}(M)$ denotes the trace of $M$. For any matrix $Z$ in $\mathbb{R}^{n p}$, the projection map $\overline{\mathrm{P}}_{U}$ on $T_{U} \mathrm{St}_{p, n}$ is given by

$$
\overline{\mathrm{P}}_{U}(Z)=Z-U \operatorname{sym}\left(U^{T} Z\right),
$$

where $\operatorname{sym}(M)=\frac{1}{2}\left(M+M^{T}\right)$. The Levi Civita connection $\bar{\nabla}$ at $U$ in $\mathrm{St}_{p, n}$ is defined, for $\eta$ in $T_{U} \mathrm{St}_{p, n}$ and $\xi$ in the set of vector fields on $\mathrm{St}_{p, n}$ denoted $\mathcal{X}\left(\mathrm{St}_{p, n}\right)$, as

$$
\bar{\nabla}_{\eta} \xi=\overline{\mathrm{P}}_{U}(\mathrm{D} \xi(U)[\eta])
$$

where $\mathrm{D} \xi(U)[\eta]$ denotes the directional derivative of $\xi$ at $U$ in the direction $\eta$. A retraction $\overline{\mathrm{R}}_{U}$ is properly defined, for all $\xi$ in $T_{U} \mathrm{St}_{p, n}$, by

$$
\overline{\mathrm{R}}_{U}(\xi)=\mathrm{qf}(U+\xi),
$$

where $\operatorname{qf}(M)$ returns the $Q$ factor of the $\mathrm{QR}$ decomposition of $M$.

\section{Special Symmetric Positive Definite Manifold}

Let $\mathcal{S}_{p}$ (respectively $\mathcal{S}_{p}^{++}$) denotes the manifold of symmetric (respectively symmetric positive definite) matrices. We define here the Special Symmetric Positive Definite manifold $\mathcal{W}_{p}$ as the set $\left\{S \in \mathcal{S}_{p}^{++}: \operatorname{det}(S)=1\right\}$.

Proposition 1. $\mathcal{W}_{p}$ is an embedded submanifold of $\mathcal{S}_{p}^{++}$of dimension $\frac{1}{2} p(p+1)-1$.

Proof. $\mathcal{W}_{p}$ is a subset of $\mathcal{S}_{p}^{++}$by definition. Consider the function $\mathrm{F}$ defined for all $S$ in $\mathcal{S}_{p}^{++}$as $\mathrm{F}(S)=\operatorname{det}(S)$.
Clearly, the image of $\mathrm{F}$ is $\mathbb{R}_{*}^{+}$and $\mathcal{W}_{p}=\mathrm{F}^{-1}(\{1\})$. Let $S$ in $\mathcal{W}_{p}$ and $X$ in $\mathbb{R}^{p p}$, the directional derivative of $\mathrm{F}$ at $S$ in the direction of $X$ is $\operatorname{DF}(S)[X]=\operatorname{tr}\left(S^{-1} X\right)$. Let $x$ in $\mathbb{R}_{*}^{+}$, then $X=\frac{x}{p} S$ is in $\mathcal{S}_{p}^{++}$and $\operatorname{DF}(S)[X]=x$. This shows that $\mathrm{F}$ is a submersion. Proposition 3.3.3 in [8] completes the proof.

\section{Proposition 2. $\mathcal{W}_{p}$ is a connected manifold.}

Proof. Consider the geodesic of $\mathcal{S}_{p}^{++}$defined for all $S_{1}$ and $S_{2}$ such that for all $t$ in $[0,1], \gamma(t)=S_{1}^{1 / 2}\left(S_{1}^{-1 / 2} S_{2} S_{1}^{-1 / 2}\right)^{t} S_{1}^{1 / 2}$ [10], where $(\cdot)^{t}$ is the power $t$. If $S_{1}$ and $S_{2}$ are in $\mathcal{W}_{p}$ then for all $t, \operatorname{det}(\gamma(t))=1$. This is a consequence of $\operatorname{det}(M N)=$ $\operatorname{det}(M) \operatorname{det}(N)$ and $\operatorname{det}\left(M^{t}\right)=(\operatorname{det}(M))^{t}$. It follows that for all $S_{1}$ and $S_{2}$ in $\mathcal{W}_{p}$ there exists a path in $\mathcal{W}_{p}$ that connects them.

Proposition 3. The tangent space of $\mathcal{W}_{p}$ at $S$ is $T_{S} \mathcal{W}_{p}=$ $\left\{X \in \mathcal{S}_{p}: \operatorname{tr}\left(S^{-1} X\right)=0\right\}$ endowed with the metric $\widetilde{\mathrm{g}}_{S}$ such that, for all $\xi$ and $\eta$ in $T_{S} \mathcal{W}_{p}, \widetilde{\mathrm{g}}_{S}(\xi, \eta)=\operatorname{tr}\left(S^{-1} \xi S^{-1} \eta\right)$.

Proof. First note that since $\mathcal{W}_{p}$ is an embedded submanifold of $\mathcal{S}_{p}^{++}$, it follows from Section 3.6.1 of [8] that $T_{S} \mathcal{W}_{p}$ is a subset of $T_{S} \mathcal{S}_{p}^{++}=\mathcal{S}_{p}$ [10]. Then, consider function $\mathrm{F}(S)=$ $\operatorname{det}(S)$ again. The kernel of $\mathrm{DF}(S)$ is the set $\left\{X \in \mathcal{S}_{p}\right.$ : $\left.\operatorname{tr}\left(S^{-1} X\right)=0\right\}$. Equation (3.19) in [8] allows to conclude that this corresponds to $T_{S} \mathcal{W}_{p}$. Finally, the Riemannian metric $\widetilde{\mathrm{g}}_{S}$ is inherited from $\mathcal{S}_{p}^{++}[10]$.

Proposition 4. For all $Z$ in $\mathbb{R}^{p p}$, the projection map $\widetilde{\mathrm{P}}_{S}$ on $T_{S} \mathcal{W}_{p}$ is given by $\widetilde{\mathrm{P}}_{S}(Z)=\operatorname{sym}(Z)-\frac{1}{p} \operatorname{tr}\left(S^{-1} \operatorname{sym}(Z)\right) S$

Proof. It is straightforward to check that $\widetilde{\mathrm{P}}_{S}$ verifies the properties of the projection map.

Proposition 5. The Levi Civita connection $\widetilde{\nabla}$ at $S$ in $\mathcal{W}_{p}$ is defined, for $\eta$ in $T_{S} \mathcal{W}_{p}$ and $\xi$ in $\mathcal{X}\left(\mathcal{W}_{p}\right)$, as $\widetilde{\nabla}_{\eta} \xi=$ $\widetilde{\mathrm{P}}_{S}\left(\mathrm{D} \xi(S)[\eta]-\operatorname{sym}\left(\eta S^{-1} \xi\right)\right)$.

Proof. Since $\mathcal{W}_{p}$ is a submanifold of $\mathcal{S}_{p}^{++}$, it follows from Proposition 5.3.2 in [8] that $\widetilde{\nabla}_{\eta} \xi=\widetilde{\mathrm{P}}_{S}\left(\widehat{\nabla}_{\eta} \xi\right)$ where $\widehat{\nabla}$ is the Levi Civita connection of $\mathcal{S}_{p}^{++}$. Using the expression of $\widehat{\nabla}$ that can be found in Appendix B of [11] completes the proof.

Proposition 6. A retraction $\widetilde{\mathrm{R}}_{S}$ is properly defined, for all $\xi$ in $T_{S} \mathcal{W}_{p}$, by $\widetilde{\mathrm{R}}_{S}(\xi)=S^{1 / 2} \exp \left(S^{-1 / 2} \xi S^{-1 / 2}\right) S^{1 / 2}$.

Proof. $\widetilde{\mathrm{R}}_{S}$ defines a retraction on $\mathcal{S}_{p}^{++}$. Furthermore, if $\operatorname{det}(S)=1$, then $\operatorname{det}\left(\widetilde{\mathrm{R}}_{S}(\xi)\right)=1$ for any $\xi$ in $T_{S} \mathcal{W}_{p}$. This comes from the fact that $\operatorname{det}(\exp (M))=\exp (\operatorname{tr}(M))$ and $\operatorname{tr}(M N)=\operatorname{tr}(N M)$. This is enough to conclude.

\section{Special Polar Manifold}

We are finally ready to define the manifold of interest. Let us name $\mathcal{M}_{p, n}=\mathrm{St}_{p, n} \times \mathcal{W}_{p}$ the Special Polar manifold. First, we will investigate the link between $\mathcal{M}_{p, n}$ and $\mathcal{B}_{p, n}$.

Proposition 7. The mapping $\pi:(U, S) \mapsto U S$ is a one to one correspondance between the elements of $\mathcal{M}_{p, n}$ and those of $\mathcal{B}_{p, n}$ (usually denoted $\mathcal{M}_{p, n} \simeq \mathcal{B}_{p, n}$ ). 
Proof. The polar decomposition of $B$ in $\mathcal{B}_{p, n}$ can be uniquely defined such that $B=U S$ where $U$ is in $\mathrm{St}_{p, n}$ and $S$ is in $\mathcal{S}_{p}^{++}$. One can show that since $\operatorname{det}(S)>0, \operatorname{det}\left(B^{T} B\right)=1$ if and only if $\operatorname{det}(S)=1$. This shows that the mapping $\pi$ defined from $\mathcal{M}_{p, n}$ to $\mathcal{B}_{p, n}$ is bijective.

As it is a product of Riemannian manifolds, $\mathcal{M}_{p, n}$ is a Riemannian manifold [8]. Due to this fact, the proofs of the following are immediate. The tangent space of $\mathcal{M}_{p, n}$ at $B=$ $(U, S)$ is $T_{B} \mathcal{M}_{p, n}=T_{U} \mathrm{St}_{p, n} \times T_{S} \mathcal{W}_{p}$ endowed with the metric $\mathrm{g}_{B}$ such that, for all $\xi=\left(\xi_{U}, \xi_{S}\right)$ and $\eta=\left(\eta_{U}, \eta_{S}\right)$ in $T_{B} \mathcal{M}_{p, n}$,

$$
\mathrm{g}_{B}(\xi, \eta)=\overline{\mathrm{g}}_{U}\left(\xi_{U}, \eta_{U}\right)+\widetilde{\mathrm{g}}_{S}\left(\xi_{S}, \eta_{S}\right) .
$$

For all $Z=\left(Z_{U}, Z_{S}\right)$ in $\mathbb{R}^{n p} \times \mathbb{R}^{p p}$, the projection map $\mathrm{P}_{B}$ at $B=(U, S)$ on $T_{B} \mathcal{M}_{p, n}$ is given by

$$
\mathrm{P}_{B}(Z)=\left(\overline{\mathrm{P}}_{U}\left(Z_{U}\right), \widetilde{\mathrm{P}}_{S}\left(Z_{S}\right)\right) .
$$

The Levi Civita connection $\nabla$ at $(U, S)$ in $\mathcal{M}_{p, n}$ is defined, for $\eta=\left(\eta_{U}, \eta_{S}\right)$ and $\xi=\left(\xi_{U}, \xi_{S}\right)$, as

$$
\nabla_{\eta} \xi=\left(\bar{\nabla}_{\eta_{U}} \xi_{U}, \widetilde{\nabla}_{\eta_{S}} \xi_{S}\right)
$$

A retraction $\mathrm{R}_{B}$ at $B=(U, S)$ is defined, for all $\xi=\left(\xi_{U}, \xi_{S}\right)$, by

$$
\mathrm{R}_{B}(\xi)=\left(\overline{\mathrm{R}}_{U}\left(\xi_{U}\right), \widetilde{\mathrm{R}}_{S}\left(\xi_{S}\right)\right)
$$

\section{E. The Objective Function on $\mathcal{M}_{p, n}$}

In order to perform AJD on $\mathcal{M}_{p, n}$ within the Riemannian framework, we need to define the objective function along with its Riemannian gradient and Hessian on the manifold.

1) Objective Funtion: Using the mapping $\pi$ defined in the previous section, one can easily find that the cost function $\mathrm{f}$ on $\mathcal{M}_{p, n}$ corresponding to (1) is defined as

$$
\begin{aligned}
\mathrm{f}(U, S) & =\sum_{k=1}^{K}\left\|\operatorname{off}\left((U S)^{T} C_{k} U S\right)\right\|_{F}^{2} \\
& =\sum_{k=1}^{K} \operatorname{tr}\left(\operatorname{off}\left((U S)^{T} C_{k} U S\right)(U S)^{T} C_{k} U S\right) .
\end{aligned}
$$

Let $\hat{\mathrm{f}}$ denotes the same function defined on $\mathbb{R}^{n p} \times \mathbb{R}^{p p}$ rather than on $\mathcal{M}_{p, n}$. $\mathrm{f}$ is then the restriction of $\hat{\mathrm{f}}$ to $\mathcal{M}_{p, n}$. This latter function $\hat{f}$ will be useful to obtain the gradient and the Hessian of $\mathrm{f}$.

2) Gradient: The gradient of $\mathrm{f}$ at $B=(U, S)$ in $\mathcal{M}_{p, n}$, denoted $\operatorname{grad} \mathrm{f}(B)$, corresponds to the only element of $T_{B} \mathcal{M}_{p, n}$ such that for all $\xi$ in $T_{B} \mathcal{M}_{p, n}, \mathrm{~g}_{B}(\operatorname{grad} \mathrm{f}(B), \xi)=\operatorname{Df}(B)[\xi]$ [8].

In practice, it is simpler to find $\operatorname{grad} \hat{\mathrm{f}}(B)$, the gradient of $\hat{\mathrm{f}}$ at $B=(U, S)$ in $\mathbb{R}^{n p} \times \mathbb{R}^{p p}$ and then deduce $\operatorname{grad} \mathrm{f}(B)$. Basic calculations lead to

$$
\begin{aligned}
\mathrm{D} \hat{\mathrm{f}}(B)[\xi] & =\sum_{k=1}^{K} 4 \operatorname{tr}\left(\xi_{U}^{T} C_{k} U S \operatorname{off}\left((U S)^{T} C_{k} U S\right) S^{T}\right) \\
& +\sum_{k=1}^{K} 4 \operatorname{tr}\left(\xi_{S}^{T} U^{T} C_{k} U S \operatorname{off}\left((U S)^{T} C_{k} U S\right)\right) .
\end{aligned}
$$

It follows that

$$
\begin{aligned}
\operatorname{grad} \hat{\mathrm{f}}(B)= & \left(\sum_{k=1}^{K} 4 C_{k} U S \operatorname{off}\left((U S)^{T} C_{k} U S\right) S^{T},\right. \\
& \left.\sum_{k=1}^{K} 4 U^{T} C_{k} U S \operatorname{off}\left((U S)^{T} C_{k} U S\right)\right) .
\end{aligned}
$$

Finally, by denoting $\operatorname{grad} \hat{\mathrm{f}}(U)$ (respectively $\operatorname{grad} \hat{\mathrm{f}}(S)$ ) the first (respectively the second) component of $\operatorname{grad} \hat{f}(B)$, one can check that $\operatorname{grad} f(B)$ is properly defined as

$$
\begin{aligned}
\operatorname{grad} \mathrm{f}(B)= & \left(\overline{\mathrm{P}}_{U}(\operatorname{grad} \hat{\mathrm{f}}(U)),\right. \\
& \left.\widetilde{\mathrm{P}}_{S}(S \operatorname{sym}(\operatorname{grad} \hat{\mathrm{f}}(S)) S)\right) .
\end{aligned}
$$

3) Hessian: The Riemannian Hessian [8] of $\mathrm{f}$ at $B$ in $\mathcal{M}_{p, n}$ is the linear mapping defined for all $\xi=\left(\xi_{U}, \xi_{S}\right)$ in $T_{B} \mathcal{M}_{p, n}$ by Hess $\mathrm{f}(B)[\xi]=\nabla_{\xi} \operatorname{grad} \mathrm{f}$, which is still in $T_{B} \mathcal{M}_{p, n}$.

Here again it is possible to find $\operatorname{Hess} \hat{\mathrm{f}}(B)$ and then deduce Hess $\mathrm{f}(B)$. One can show that Hess $\hat{\mathrm{f}}(B)$ is given by

$$
\begin{aligned}
& \text { Hess } \hat{\mathrm{f}}(B)[Z]=\mathrm{D} \operatorname{grad} \hat{\mathrm{f}}(B)[Z]= \\
& \left(\sum_{k=1}^{K} 4 C_{k}\left(\dot{V} M_{k} S^{T}+V \dot{M}_{k} S^{T}+V M_{k} Z_{S}^{T}\right),\right. \\
& \left.\sum_{k=1}^{K} 4 Z_{U}^{T} C_{k} V M_{k}+4 U^{T} C_{k}\left(\dot{V} M_{k}+V \dot{M}_{k}\right)\right),
\end{aligned}
$$

where $Z=\left(Z_{U}, Z_{S}\right)$ is in $\mathbb{R}^{n p} \times \mathbb{R}^{p p}, V=U S, \dot{V}=$ $Z_{U} S+U Z_{S}, M_{k}=\operatorname{off}\left(V^{T} C_{k} V\right)$ and $\dot{M}_{k}=\operatorname{off}\left(\dot{V}^{T} C_{k} V+\right.$ $\left.V^{T} C_{k} \dot{V}\right)$.

The first (respectively second) component of $\operatorname{Hess} f(B)[\xi]$ is denoted $\operatorname{Hess} \mathrm{f}(U)[\xi]$ (respectively $\operatorname{Hess} \mathrm{f}(S)[\xi]$ ) where $\xi$ is in $T_{B} \mathcal{M}_{p, n}$. The same notations are used for $\hat{f}$. It follows that

$$
\begin{aligned}
\operatorname{Hessf}(U)[\xi] & =\overline{\mathrm{P}}_{U}(\operatorname{Hess} \hat{\mathrm{f}}(U)[\xi]) \\
& -\overline{\mathrm{P}}_{U}\left(\xi_{U} \operatorname{sym}\left(U^{T} \operatorname{grad} \hat{\mathrm{f}}(U)\right)\right) \\
\operatorname{Hessf}(S)[\xi] & =\widetilde{\mathrm{P}}_{S}(S \operatorname{sym}(\operatorname{Hess} \hat{\mathrm{f}}(S)[\xi]) S) \\
& +2 \widetilde{\mathrm{P}}_{S}\left(\xi_{S} \operatorname{sym}(\operatorname{grad} \hat{\mathrm{f}}(S)) S\right) \\
& +\widetilde{\mathrm{P}}_{S}\left(\mathrm{D} \widetilde{\mathrm{P}}_{S}\left[\xi_{S}\right](S \operatorname{sym}(\operatorname{grad} \hat{\mathrm{f}}(S)) S)\right) \\
& -\widetilde{\mathrm{P}}_{S}\left(\operatorname{sym}\left(\xi_{S} S^{-1} \operatorname{grad}(S)\right)\right),
\end{aligned}
$$

where for all $Z_{s}$ in $\mathbb{R}^{p p}$ and $\xi_{S}$ in $T_{S} \mathcal{W}_{p}$

$$
\begin{aligned}
\mathrm{D} \widetilde{\mathrm{P}}_{S}\left[\xi_{S}\right]\left(Z_{S}\right) & =\frac{1}{p} \operatorname{tr}\left(S^{-1} \xi_{S} S^{-1} \operatorname{sym}\left(Z_{S}\right)\right) S \\
& -\frac{1}{p} \operatorname{tr}\left(S^{-1} \operatorname{sym}\left(Z_{S}\right)\right) \xi_{S} .
\end{aligned}
$$

\section{F. The Trust-Region Method}

We will minimize $\mathrm{f}$ over the manifold $\mathcal{M}_{p, n}$ by a standard second-order Riemannian optimization algorithm, the Riemmannian trust-region method [8]. We sketch here the main idea of this algorithm and refer to Chapter 7 in [8] for more details. Starting from an initial point $B_{0}$ in $\mathcal{M}_{p, n}$, a sequence of iterates $\left\{B_{l}\right\}$ is computed in order to find a minimizer to the cost function $\mathrm{f}$. To do so, a model $\mathrm{m}_{B_{l}}$ of $\mathrm{f}$ around the iterate $B_{l}$ is constructed using the second-order Taylor expansion for $\xi$ in $T_{B_{l}} \mathcal{M}_{p, n}$ such as

$$
\begin{array}{r}
\mathrm{m}_{B_{l}}(\xi)=\mathrm{f}\left(B_{l}\right)+\mathrm{g}_{B_{l}}\left(\operatorname{grad} \mathrm{f}\left(B_{l}\right), \xi\right) \\
+\frac{1}{2} \mathrm{~g}_{B_{l}}\left(\operatorname{Hessf}\left(B_{l}\right)[\xi], \xi\right) .
\end{array}
$$


This model is considered to be valid in a trust-region defined as a ball in $T_{B_{l}} \mathcal{M}_{p, n}$ with radius $\Delta_{l}$. It is then needed to find the solution $\xi_{l}$ to the subproblem of minimizing $\mathrm{m}_{B_{l}}$ within the ball of radius $\Delta_{l}$ in the tangent space of $B_{l}$. This is done using the truncated conjugate-gradient method. The candidate next iterate is then $\mathrm{R}_{B_{l}}\left(\xi_{l}\right)$. This candidate is evaluated to check that the model $\mathrm{m}_{B_{l}}$ at $\xi_{l}$ is valid. If it is the case, the candidate is accepted and the radius $\Delta_{l}$ can even be increased if the model is very good. Otherwise, the candidate is rejected and $\Delta_{l}$ is decreased. The stopping criterion is defined by the norm of the Riemannian gradient lower than a tolerance (fixed at $10^{-5}$ in our numerical experiments).

\section{RESULTS}

In order to estimate the perfomances of our method, named RAJD (for Riemannian AJD), we simulated data and compared it to NoJOB [2] and UWEDGE [3]. RAJD was performed using manopt toolbox [12] in Matlab (c) with modifications in order to use the new manifold defined here.

To analyze how the methods behaved, we used two criteria. The first one is the Moreau-Amari index $I_{\mathrm{M}-\mathrm{A}}$ [13] defined as

$$
\begin{array}{r}
I_{\mathrm{M}-\mathrm{A}}=\frac{1}{2 n(n-1)}\left(\sum_{i=1}^{n}\left[\frac{\sum_{j=1}^{n}\left|H_{i j}\right|}{\max _{1 \leq j \leq n}\left|H_{i j}\right|}-1\right]\right. \\
\left.+\sum_{j=1}^{n}\left[\frac{\sum_{i=1}^{n}\left|H_{i j}\right|}{\max _{1 \leq i \leq n}\left|H_{i j}\right|}-1\right]\right)
\end{array}
$$

where $H=B^{T} A$, with $B$ the estimated unmixing matrix and $A$ the true mixing matrix. The second one is a measure of non-diagonality $I_{\mathrm{n}-\mathrm{d}}$ of the transformed dataset defined as

$$
I_{\mathrm{n}-\mathrm{d}}=\frac{1}{K(n-1)} \sum_{k=1}^{K} \frac{\left\|\operatorname{off}\left(B^{T} C_{k} B\right)\right\|_{F}^{2}}{\left\|\operatorname{diag}\left(B^{T} C_{k} B\right)\right\|_{F}^{2}}
$$

where $\operatorname{diag}(M)$ denotes the diagonal part of the matrix $M$.

We performed 200 tests. For each test, as it is done in [3], we generated $K=100$ matrices $\left\{C_{k}\right\}_{1 \leq k \leq K}$ of dimension $n \times n$ according to

$$
C_{k}=A D_{k} A^{T}+\frac{1}{2 \sigma}\left(N_{k}+N_{k}^{T}\right)
$$

where $A$ is a non-orthogonal mixing matrix with i.i.d. elements generated from a normal distribution $\mathcal{N}(0,1)$. We controlled the non-orthogonality by constraining the condition number with respect to inversion between 3 and 7 . Matrices $\left\{D_{k}\right\}_{1 \leq k \leq K}$ are diagonal with i.i.d. elements generated from a chi-squared distribution corresponding to the power of the sources in the BSS problem. $\sigma$ is a free parameter defining the noise level and $N_{k}$ is a noise matrix with elements drawn independently from a normal distribution $\mathcal{N}(0,1)$. No dimension reduction was performed (i.e., $p=n$ ) and all algorithms were initialized with the identity.

In figure 1, the Moreau-Amari index $I_{\mathrm{M}-\mathrm{A}}$ of the three methods for $n=8$ as a function of the noise parameter $\sigma$ is presented. One can see that all three methods have similar behaviours even though UWEDGE gives, in general, results

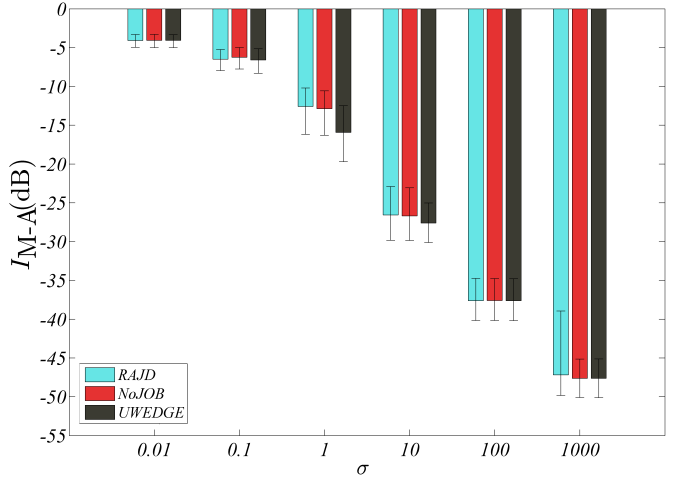

Fig. 1. Median and quantiles (5\% and 95\%) over 200 tests of the MoreauAmari index as a function of the noise parameter $\sigma$ for the three methods for $n=8$. All three methods have comparable performance. UWEDGE have slightly better results. See text for details.

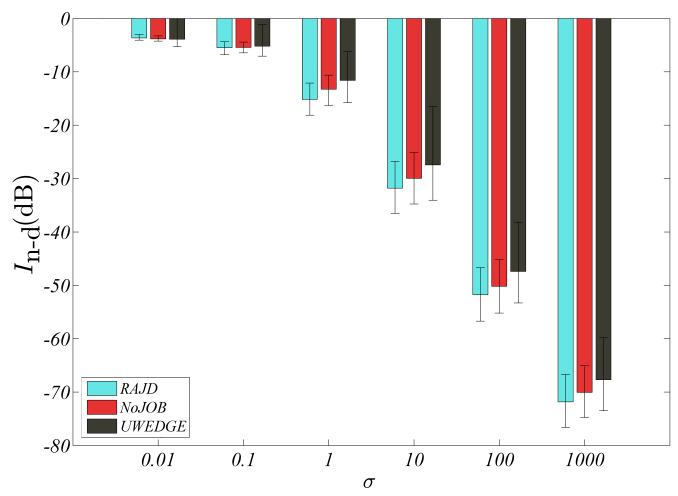

Fig. 2. Median and quantiles (5\% and $95 \%$ ) over 200 tests of the nondiagonality measure as a function of the noise parameter $\sigma$ for the three methods for $n=8$. Again, all three methods have comparable results. RAJD diagonalizes the datasets better than the other methods. See text for details.

slightly closer to the true solution. In figure 2, we plotted the non diagonality measure $I_{\mathrm{n}-\mathrm{d}}$ as a function of the noise parameter $\sigma$ for all methods for $n=8$. It can be noticed that here RAJD diagonalized the datasets better. Even though RAJD and NoJOB succeeded better in diagonalizing the datasets, the solutions found by UWEDGE are generally closer to the true solutions. This reflects the fact that when noise is added, the true solution does not necessarily correspond to the one that diagonalizes the dataset better.

In figures 3 and 4, we studied the effect of the dimension $n$ of the matrices $C_{k}$ for the three methods for $\sigma=100$. As expected, the results deteriorate when $n$ increases for all methods. Moreover, we still have comparable performance for all methods and it is consistent with results obtained when the influence of the noise was studied. Indeed, the joint diagonalizer of UWEDGE is generally slightly closer to the true solution (see figure 3 ) and RAJD diagonalizes the datasets better (figure 4). This shows that RAJD is still a valid method when the dimension of the problem is increased.

RAJD and NOJoB use the same cost function (1) but the cost function of UWEDGE is slightly different [3]. Our results 


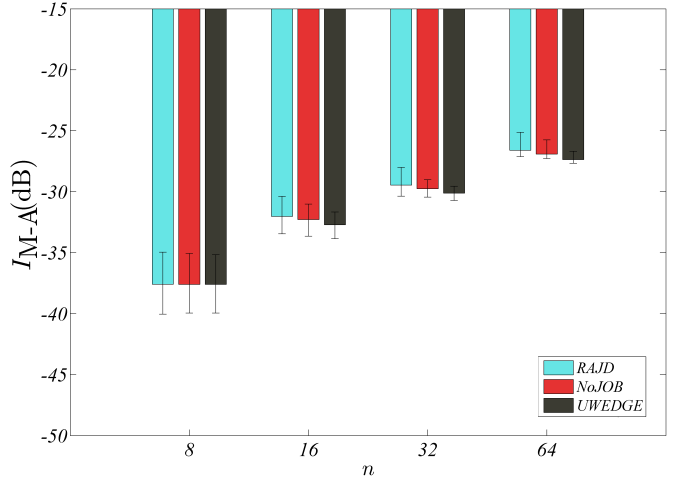

Fig. 3. Median and quantiles (5\% and 95\%) over 200 tests of the MoreauAmari index as a function of matrices dimension $n$ for the three methods for $\sigma=100$. The performance decreases when the dimension increases. All methods have comparable performances. See text for details.

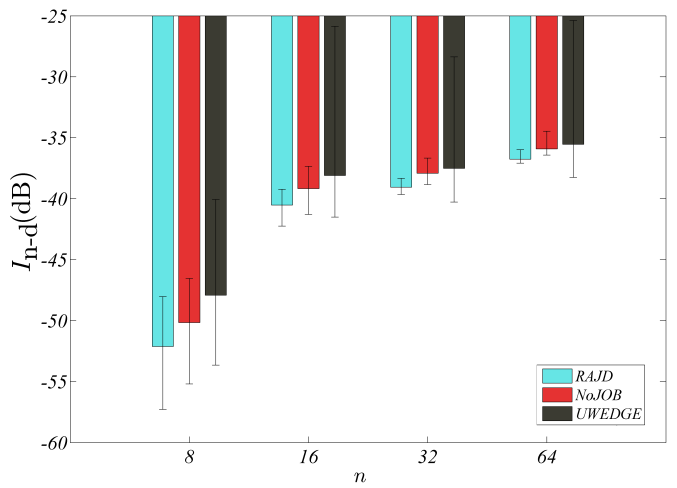

Fig. 4. Median and quantiles (5\% and 95\%) over 200 tests of the nondiagonality measure as a function of matrices dimension $n$ for the three methods for $\sigma=100$. The performance decreases when the dimension increases. All methods have comparable performances. See text for details.

suggest that objective function (1) leads to an overfitting of the data. This indicates that the Riemannian method that we considered is efficient in minimizing (1) but this function seems not to be well suited for our purpose. Furthermore, the fact that RAJD gave better results for $I_{\mathrm{n}-\mathrm{d}}$ than NOJoB, which uses the same cost function, suggest that Riemannian optimization on the special polar manifold is a promising tool. The use of a more appropriate cost function may lead to better results as compared to state of the art methods such as UWEDGE.

\section{CONCLUSION}

In this article we have properly defined the special polar manifold allowing the investigation of the AJD problem from a Riemannian optimization point of view. Of course, this new manifold may turn useful in many other problems. The results we obtained for the AJD problem are encouraging and the limits encountered seems to be related to objective function we have considered. Other cost functions such as the ones in [3] or [14] are under consideration and will be presented in future works. Other Riemannian optimization algorithms can also be investigated. Note that since accuracy is sought here, second order algorithms (using Riemannian Hessian or approximation of it) should be preferred. We are also interested in studying direct dimension reduction allowing to avoid the usual whitening step, with possible gain in precision.

This study shows that working within the Riemannian geometry framework is appropriate to solve the AJD problem. Furthermore, unlike the strategies discussed in the introduction, this approach allows to easily change the optimization scheme or the cost function, as long as this latter is smooth. Other related models can also simply be considered such as extensions to block AJD or joint BSS [7].

\section{ACKNOWLEDGMENT}

The authors would like to thank Bijan Afsari for his helpful comments on this work. This work has been partially supported by the LabEx PERSYVAL-Lab (ANR-11-LABX-002501) funded by the French program "Investissement d'avenir" and the European Research Council project CHESS 2012ERC-AdG-320684.

\section{REFERENCES}

[1] P. Comon and C. Jutten. Handbook of Blind Source Separation: Independent Component Analysis and Applications. Academic Press, 1st edition, 2010.

[2] M. Congedo, R. Phlypo, and J. Chatel-Goldman. Orthogonal and nonorthogonal joint blind source separation in the least-squares sense. In 20th European Signal Processing Conference (EUSIPCO-2012), pages 1885-1889, 2012.

[3] P. Tichavskỳ and A. Yeredor. Fast approximate joint diagonalization incorporating weight matrices. Signal Processing, IEEE Transactions on, 57(3):878-891, 2009.

[4] P.-A. Absil and K.A. Gallivan. Joint diagonalization on the oblique manifold for independent component analysis. In Acoustics, Speech and Signal Processing, 2006. ICASSP 2006 Proceedings. 2006 IEEE International Conference on, volume 5, pages V-V, May 2006.

[5] B. Afsari and P. S. Krishnaprasad. Some gradient based joint diagonalization methods for ICA. In Independent Component Analysis and Blind Signal Separation, pages 437-444. Springer, 2004.

[6] F. J. Theis, T. P. Cason, and P.-A. Absil. Soft dimension reduction for ICA by joint diagonalization on the Stiefel manifold. In Independent Component Analysis and Signal Separation, pages 354-361. Springer, 2009.

[7] M. Congedo. EEG Source Analysis. CNRS, University of Grenoble Alpes, Grenoble Institute of Technology, 2013.

[8] P.-A. Absil, R. Mahony, and R. Sepulchre. Optimization Algorithms on Matrix Manifolds. Princeton University Press, Princeton, NJ, USA, 2008.

[9] M. Congedo, B. Afsari, A. Barachant, and M. Moakher. Approximate joint diagonalization and geometric mean of symmetric positive definite matrices. PLoS ONE, 10(4):e0121423, 042015.

[10] R. Bhatia. Positive definite matrices. Princeton University Press, 2009.

[11] G. Meyer. Geometric optimization algorithms for linear regression on fixed-rank matrices. PhD thesis, University of Liège, 2011.

[12] N. Boumal, B. Mishra, P.-A. Absil, and R. Sepulchre. Manopt, a Matlab toolbox for optimization on manifolds. Journal of Machine Learning Research, 15:1455-1459, 2014

[13] E. Moreau and O. Macchi. New self-adaptative algorithms for source separation based on contrast functions. In Higher-Order Statistics, 1993., IEEE Signal Processing Workshop on, pages 215-219. IEEE, 1993.

[14] D.-T. Pham. Joint approximate diagonalization of positive definite 2000. 